\title{
PROMÉXICO: UNA EXPERIENCIA PÚBLICO-PRIVADA EXITOSA. SU ORIGEN, CONSOLIDACIÓN, DESAPARICIÓN Y POTENCIAL REIMPLANTACIÓN.
}

\section{PROMEXICO: A SUCCESSFUL PUBLIC-PRIVATE EXPERIENCE. ORIGIN, CONSOLIDATION, DISAPPEARANCE AND POTENTIAL REIMPLANTATION}

Mtro. Luis Manuel Cuevas Padilla ${ }^{\text {a }}$

Dr. René Mariani Ochoa ${ }^{b}$

${ }^{a}$ Universidad Veracruzana Instituto de Investigaciones y Estudios Superiores de la Ciencias Administrativas, luicuevas@uv.mx

${ }^{\mathrm{b}}$ Universidad Veracruzana Instituto de Investigaciones en Contaduría, rmariani@uv.mx

\section{RESUMEN}

ProMéxico, la agencia del Gobierno de México de promoción empresarial a nivel internacional, funcionó con gran éxito de 2007 a 2018. En el artículo se comenta su origen, su desarrollo y consolidación como agencia de promoción internacional, su abrupto cierre y las consecuencias de ello. Se hace énfasis en los mecanismos de operación que tenía la institución, así como los resultados obtenidos durante su existencia.

La metodología empleada es un análisis documental sobre los antecedentes y operación de la institución, apoyado con estadísticas de fuentes oficiales y estructurado con base a la experiencia de los autores. 
En la parte final se presentan conclusiones enfocadas en ver en la desaparición de ProMéxico no tanto los efectos que ya se están percibiendo en las tendencias de exportaciones e inversión extranjera en México, sino en la necesidad de diseñar estrategias de promoción económica internacional que ayuden a retomar el crecimiento de la economía externa del país.

PALABRAS CLAVE: ProMéxico; Exportación; Inversión extranjera; Internacionalización.

\section{ABSTRACT}

ProMéxico was the international promotion agency of the Government of Mexico and operated with great success from 2007 to 2018. The article discusses its origin, its development and consolidation as an international promotion agency, its abrupt closure and the consequences of it. Emphasis is making on the operating mechanisms that the institution had, as well as the results obtained during its existence.

The methodology used is a documentary analysis of the background and operation of the institution, supported with statistics from official sources and structured with the experience of the authors.

In the final part, conclusions are presented with focus on seeing in the disappearance of ProMéxico not so in much the effects that already are being perceived in the trends of exports and foreign investment in Mexico, rather in the need to design international economic promotion strategies that help to resume the growth of the country's external economy.

KEYWORDS: ProMéxico; export; foreign investment; internationalization.

\section{INTRODUCCIÓN}

La promoción de los negocios internacionales de un país tan relevante en el comercio exterior como México no debería estar sujeta a esfuerzos aislados de diferentes actores, que si bien son importantes, lo serían más aún con una estrategia de promoción de 
exportaciones e inversión, muy bien analizada, de largo plazo, y en la que, para reducir el gasto para el gobierno federal, los gobiernos locales y el sector privado jugaran un papel importante, pero con procedimientos, canales de comunicación y metas muy bien definidos.

El caso de ProMéxico muestra claramente como una organización con políticas, procedimientos y objetivos claros, así como un capital humano bien preparado, puede dar grandes beneficios a un país, sin necesidad de enormes estructuras.

Sin duda, la historia de ProMéxico puede servir de ejemplo a países que le apuesten a la competitividad internacional como estrategia de desarrollo, y probablemente para un futuro rediseño de la política pública en México, orientada hacia la atracción de inversiones productivas y apertura de nuevos mercados externos para los productos nacionales.

En este contexto, la conveniencia del presente trabajo radica en cubrir una necesidad de información para que los interesados cuenten con un documento de análisis que contribuya a la contextualización de investigaciones y estudios en materia de políticas públicas sobre promoción económica internacional tanto en México, como en países que pasen proceso de cambio similares a este país.

El artículo tiene como objetivo general dar a conocer al lector el origen de ProMéxico, su desarrollo como agencia de promoción internacional, así como analizar su cierre, consecuencias y posibles soluciones.

Por su parte los objetivos específicos que se tienen son conocer los antecedentes y causas que dieron origen a la creación de ProMéxico, los logros que alcanzó la institución en su consolidación; analizar el proceso con el que se dio su extinción, las consecuencias que se están teniendo en México con su desaparición, así como identificar posibles soluciones de reimplantación.

\section{ANTECEDENTES DEL COMERCIO EXTERIOR EN MÉXICO}

El comercio internacional en México nació en el siglo XVI, prácticamente desde el inicio del virreinato, con el desarrollo de la ruta conocida como la Nao de China. Mediante esta ruta marítima-terrestre, las mercancías del lejano oriente, principalmente de China y Japón, 
se concentraban en Manila, Filipinas, y de ahí se embarcaban en un tipo de barco mercante, conocido como nao, para llegar al Puerto de Acapulco. Posteriormente se transportaban por tierra al Puerto de Veracruz, para ser embarcadas desde ahí con destino a los puertos españoles en Europa, retornando las naves a Veracruz, con mercancías europeas destinadas a la Nueva España y otras que llegaban hasta el lejano oriente, haciendo la ruta inversa.

Con el paso de los siglos la ruta y sus buques se fueron modernizando, constituyéndose probablemente en la ruta comercial más importante del mundo de su tiempo, al grado de que el peso mexicano de plata, usado para las transacciones comerciales en la ruta, se consolidó como la moneda más fuerte y utilizada de esa época.

Sin embargo, con la independencia de México de España, la ruta se interrumpió bruscamente y el comercio internacional de México se redujo a niveles sumamente bajos, con algunos repuntes como el originado por la construcción del puerto artificial de Veracruz a finales del porfiriato, o largos periodos de estancamientos para el comercio exterior por modelos económicos como el de substitución de importaciones en las décadas de la postguerra, ya en el Siglo XX.

La promoción de la inversión y los negocios internacionales en México tiene su fundamento jurídico en la propia Constitución Política de los Estados Unidos Mexicanos, que en su artículo 25 señala que "corresponde al Estado Mexicano garantizar el fomento del crecimiento económico mediante la competitividad, entendida esta como el conjunto de condiciones necesarias para generar un mayor crecimiento económico, promoviendo la inversión y la generación de empleo" (CPEUM, Art. 25).

El primer antecedente de un organismo público del Gobierno de México dedicado a la promoción de la exportación de productos mexicanos, fue la creación del Banco Nacional de Comercio Exterior (BANCOMEXT) en 1937, aunque al ser una institución dedicada inherentemente a las actividades financieras, las actividades de promoción internacional estaban en un segundo plano.

Fue hasta diciembre de 1970, todavía en plena operación del modelo de substitución de importaciones, que se creó el Instituto Mexicano de Comercio Exterior (IMCE), (Ley que 
crea el Instituto Mexicano de Comercio Exterior, 1970) con funciones muy específicas para promover la presencia de productos de México en el exterior, similares a cualquier agencia de promoción internacional de la actualidad, tomando para sí las que realizaba en forma incipiente BANCOMEXT.

Extrañamente fue un fenómeno natural y no una política pública lo que provocó el cierre del IMCE. El trágico terremoto de septiembre de 1985, que afectó la Ciudad de México, causó el derrumbe de muchos grandes edificios, con la lamentable pérdida de gran número de vidas. Uno de ellos fue el edificio principal de la Secretaría de Comercio y Fomento Industrial (SECOFI) del Gobierno Federal y, al quedarse la SECOFI sin su sede principal, traslado su oficina corporativa al edificio que ocupaba el IMCE en la colonia Hipódromo Condesa de la capital del país, mismo que ocupa hasta la fecha, aunque ya con el nombre de Secretaría de Economía.

Al quedarse el IMCE sin oficinas, por cedérselas a la SECOFI, la solución que encontró la administración del presidente Miguel De la Madrid fue simple, aunque probablemente no la más sensata: desaparecer al IMCE y pasar sus funciones integras a BANCOMEXT, que retomaría actividades que ya había realizado en forma básica en sus inicios. Todo esto se dio justamente en el año previo a la entrada de México al GATT, en el momento en que más necesitaba el país un organismo dedicado a la promoción de exportaciones para aprovechar ese hecho que marcó la apertura comercial de México hacia el mundo.

Por otra parte, para atraer inversión extranjera hacia México, se creó en 1989 el Consejo Mexicano de Inversión (CMI), mejor conocido por su nombre en inglés Mexican Investment Board (MIB), organismo público-privado destinado a apoyar a inversionistas extranjeros a explorar y concretar oportunidades de inversión en México (De la Mora, 2017, p. 19). El MIB funcionó intensamente durante la oleada de privatizaciones de la década de los 90’s, y desapareció en el año 2000, ante el poco interés del sector privado y del Gobierno Federal para seguirlo financiando.

La creación de agencias gubernamentales para la promoción internacional fue una tendencia a nivel mundial que se empezó a dar desde los años 70’s, con casos muy exitosos 
en Hipanoamérica, como ProChile, fundado en 1974 en Chile; el ICEX de España, abierto en 1982; ProExport, la agencia colombiana creada en 1991 y conocida actualmente como ProColombia; ProComer, agencia de Costa Rica fundada en 1996, y más recientemente PromPerú en 2007.

Son de llamar la atención dos agencias de promoción internacional creadas en México, no por el Gobierno Federal, sino por gobiernos estatales, y que han obtenidos resultados relevantes en el fomento de exportaciones y atracción de inversiones hacia sus entidades. La primera, la Coordinadora de Fomento al Comercio Exterior (COFOCE) de Guanajuato, creada desde 1992 y funcionando eficazmente hasta la actualidad, y la segunda, Jaltrade en Jalisco, creado en 1999, y que después de un muy buen desempeño durante casi 20 años, dejó de ser un organismo público descentralizado y su estructura y funciones fueron absorbidas recientemente por la Secretaría de Desarrollo Económico de ese estado.

\section{1 ProMéxico}

A nivel nacional tuvieron que pasar más de 20 años para que México pudiera volver a contar con un organismo dedicado específicamente a la promoción internacional, hasta que, mediante un decreto del entonces presidente Felipe Calderón en junio de 2007 (Decreto por el que se ordena la constitución del Fideicomiso Público considerado Entidad Paraestatal denominado ProMéxico, 2007), se fundó ProMéxico, tomando como ejemplo los casos exitosos de otras agencias internacionales en Latinoamérica ya consolidadas, como ProChile (Chile), ProComer (Costa Rica) y ProExport (Colombia).

Cabe señalar que ProMéxico estuvo contemplado dentro del Plan Nacional de Desarrollo 2007-2012, dentro de su eje 3 Fomentar el Comercio Exterior y la Inversión Extranjera Directa, y en el Plan Nacional de Desarrollo 2013-2018, como parte de su estrategia 4.2 Propiciar la coordinación interinstitucional en México en materia de promoción y difusión hacia el exterior.

En un principio ProMéxico funcionó con la misma estructura humana y material con la que BANCOMEXT realizaba las actividades de promoción de exportaciones y atracción de inversión extranjera, con oficinas corporativas en la zona de San Jerónimo en la Ciudad de 
México, para después de un par de años contar con algunas oficinas estatales en los estados del país con mayor actividad económica, y representaciones en los países con los que México tenía más intercambio comercial y de inversión. Posteriormente, en su consolidación alcanzó a tener oficinas en 30 de los 32 estados y 46 consejerías comerciales en el exterior, distribuidas estratégicamente en países de los cinco continentes.

La figura jurídica con la que se fundó ProMéxico fue la de fideicomiso público, que da gran versatilidad en sus operaciones, pero expuesto a ser extinto cuando el fideicomitente lo decida, en este caso el propio Gobierno Federal.

A diferencia de BANCOMEXT, que estaba sectorizado a la Secretaría de Hacienda y Crédito Público, ProMéxico estaba sectorizado a la Secretaría de Economía, teniendo a la cabeza del organismo, como todo fideicomiso público, un comité técnico, con el Secretario de Economía en turno como presidente de dicho comité.

Esa sectorización de ProMéxico a la Secretaría de Economía está reforzada en las atribuciones que le da a esa secretaría la Ley de Comercio Exterior y la Ley de Inversión Extranjera. La Ley de Comercio Exterior, desde su artículo $1^{\circ}$ señala su objetivo de promover el comercio exterior, reforzándolo en su artículo $5^{\circ}$, al otorgarle la facultad de establecer los programas y mecanismos de promoción y fomento de las exportaciones.

Por su parte, en lo que respecta a la inversión, la Ley de Inversión Extranjera en su artículo 26, le da a dicha Secretaría, a través de la Comisión Nacional de Inversiones Extranjeras, la atribución de diseñar mecanismos para promover la inversión en México.

A la cabeza operativa de ProMéxico existía un director general, y a lo largo de su historia fueron cinco los funcionarios que desempeñaron ese puesto, desde su fundador, Bruno Ferrari, seguido por Carlos Guzmán, Ernesto De Lucas y Francisco González, siendo el último Paulo Carreño.

Muy acorde a su lema Inversión y comercio, ProMéxico nació con dos objetivos originales: promover las exportaciones de productos mexicanos y atraer inversión extranjera en sectores productivos hacia México, objetivos normales en la mayoría de las agencias de 
promoción internacional de otros países.

Un objetivo que nunca se incluyó fue la promoción de las importaciones a través del desarrollo de proveedores extranjeros confiables, que, aunque es parte de los negocios internacionales, solo está presente entre los objetivos de agencias de ciertos países con alto grado de desarrollo como Japón, Canadá y Alemania.

En 2011 se agregó un tercer objetivo a los dos ya existentes, al que se le denominó internacionalización de empresas mexicanas. Aunque el término internacionalización tiene varias acepciones, en el caso de ProMéxico se definía como el que empresas mexicanas tuvieran presencia física en el extranjero, desde una simple oficina de representación, hasta una planta productiva en otro país.

Este tercer objetivo, aunque originó muchos casos exitosos para México, nunca fue muy publicitado, ante el temor de generar la impresión de que la agencia generara empleos en el extranjero, por parte de críticos que no consideraran que la inversión mexicana en el extranjero, es en muchas ocasiones algo muy necesario para que las empresas sean competitivas en los mercados globales actuales.

Funcionalmente la estructura operativa de ProMéxico, de poco más de 500 personas (ProMéxico, 2017, Una década de promover a México en el mundo, p. 76), tenía una Dirección General a la cabeza, de la que dependían varias unidades, que, aunque tuvieron ajustes en número y funciones a lo largo del tiempo, respondía a una división lógica en base a sus actividades.

Al respecto, en su última etapa ProMéxico constó de tres unidades de procesos sustantivos: la Unidad de Promoción Sectorial, la Unidad de Inteligencia de Negocios y la Unidad de Promoción de Negocios Globales.

La Unidad de Promoción Sectorial desarrollaba estrategias de trabajo que se adaptaban a los diferentes sectores productivos en México y el mundo, mientras que la Unidad de Inteligencia de Negocios realizaba una intensa labor de búsqueda, análisis y generación de conocimientos que ayudaran a las otras unidades en sus labores. 
Por su parte, la Unidad de Promoción de Negocios Globales era la que implementaba las relaciones con las empresas mexicanas y extranjeras, promoviendo el enlazamiento entre exportadores de productos mexicanos y compradores de los mismos en el extranjero; entre inversionistas extranjeros y autoridades gubernamentales en México, así como entre inversionistas mexicanos con gobiernos extranjeros. En esa unidad estaban ubicadas tanto las 30 direcciones estatales en México, que tenían el contacto con las empresas mexicanas y gobiernos locales, como las 46 consejerías comerciales ubicadas alrededor del mundo, que a su vez mantenían la relación con los importadores, inversionistas y gobiernos extranjeros.

Finalmente, como toda organización de su tamaño, contaba con tres unidades de apoyo: la Unidad de Administración y Finanzas, la Coordinación General de Asuntos Jurídicos y la Coordinación de Comunicación Institucional. Un organigrama de dicha estructura se puede ver en el anexo 1.

ProMéxico contaba un amplio catálogo de servicios y apoyos a las empresas, diseñados para promover las exportaciones y la internacionalización de empresas mexicanas. Aunque todos eran instrumentos para ayudar a las empresas en los negocios internacionales, operativamente ProMéxico le llamaba "servicios" a las acciones que realizaba para una empresa en particular, y la empresa pagaba a ProMéxico la parte del costo del servicio que no estaba subsidiada por el gobierno federal. Este costo podía ser desde 0, hasta varios miles de pesos, aunque siempre muy inferior a lo que le costaría a la empresa ese servicio en la consultoría privada.

Cuando ProMéxico no tenía la capacidad técnica para realizar el instrumento que requería la empresa, otorgaba lo que se le conocía como apoyo, con el que daba el recurso económico a la empresa para realizar la actividad que requería, dentro de un catálogo de apoyos con conceptos y montos muy bien definido. Cabe señalar que todos los apoyos que daba ProMéxico a las empresas eran a reembolso, esto es, el dinero se le daba al solicitante hasta que hubiera realizado exitosamente la actividad para la que solicitó el apoyo, y comprobado documentalmente su realización. Esto garantizaba una aplicación al 100\% de los apoyos hacia el fin para el que eran solicitados. 
Un listado del catálogo de apoyos y servicios se presenta en el anexo 2, aunque los más utilizados y que generaron mejores resultados fueron:

- Agendas y misiones comerciales: Serie de citas organizadas por ProMéxico para un exportador específico, con compradores extranjeros interesados en sus productos.

- Agendas y misiones de inversión: Visita de representantes de gobiernos estatales al extranjero para promover las oportunidades de inversión en su entidad, o visita de inversionistas extranjeros identificados por ProMéxico, para visualizar posibles sitios para sus inversiones o reunirse con autoridades relacionadas con sus proyectos.

- Mesas de negocios. Eventos en México o el extranjero donde se reunían, en un mismo lugar y fecha, a un grupo grande de exportadores e importadores de diferentes países, que sostenían entrevistas de negocios entre sí.

- Ferias internacionales. Exposiciones internacionales de amplio prestigio en el extranjero, en las que ProMéxico contrataba un espacio extenso, llamado pabellón mexicano, donde construía stands para ponerlos a disposición de exportadores mexicanos para promover sus productos.

- Certificaciones. Apoyo a los exportadores mexicanos para que alcanzaran certificaciones sobre inocuidad, calidad, responsabilidad social, etc., cubriendo parte del costo del proceso de obtenerlas.

- Registro de marca. Apoyo a los exportadores con el costo del registro de sus marcas en otros países, con la finalidad de que las protegieran contra su uso indebido en el extranjero.

\section{2 Resultados De ProMéxico}

Con la finalidad de dar certidumbre, transparencia y seguimiento a los resultados de ProMéxico, la institución tenía la norma de que cualquier exportación o inversión que se lograra gracias a su intervención, tenía que estar soportada por una carta firmada por el 
representante legal de la empresa en la que se indicaba, para la exportación, la empresas exportadora, la empresa importadora y el monto de la venta; mientras que en el caso de la inversión el monto a invertir comprometido y los empleos a generar.

Asombran las cifras de los resultados alcanzados por ProMéxico en sus 11 años de existencia. Logró atraer inversión extranjera directa a México por más de 120,000 millones de dólares, que generaron arriba de 440,000 empleos directos, en más de 1,300 proyectos atendidos. Se calcula que alrededor de la mitad de la inversión extranjera directa en México de 2008 a 2018 llegó al país gracias a la intervención de ProMéxico.

En materia de exportación, en ese mismo periodo, con el apoyo de ProMéxico, más de 6,000 empresas mexicanas lograron exportar mercancías por un monto superior a los 19,000 millones de dólares a más de 70 países alrededor del mundo.

Por su parte, mediante la estrategia de internacionalización de empresas, cerca de 200 compañías mexicanas lograron establecer filiales propias en el extranjero para tener una presencia más directa en sus países de interés (ProMéxico, 2017, Revista Negocios ProMéxico Mayo/2017, p. 57) (ProMéxico, 2017, Una década de promover a México en el mundo, p. 10) (ProMéxico, 2018, Cartera de proyectos de exportación e inversión).

En consecuencia, cabría preguntarse: ¿acaso no valdría la pena reimplantar a ProMéxico?

Uno de los cuestionamientos fuertes que tuvo ProMéxico por parte de quienes promovieron su cierre, fue el presupuesto que invertía el Gobierno Federal en su operación, que en todos sus 11 años sumó un total de 14,145 millones de pesos. Pero si comparamos esa inversión con los resultados obtenidos, dividiendo el presupuesto total entre los flujos de exportación e inversión antes citados, convertidos a pesos mexicanos, se tuvo una rentabilidad de 28 pesos ingresados a México en divisas por exportaciones, por cada peso gastado en ProMéxico; mientras que, en inversión extranjera, cada peso gastado en ProMéxico originó que compañías extranjeras invirtieran 178 pesos en México. En total, una relación de 206 a 1. Difícilmente una inversión puede dar esos resultados. Los cálculos para estimar la rentabilidad se muestran en el anexo 3, mientras que los resúmenes de las cuentas públicas de ProMéxico se incluyen en el anexo 4. 
Es de notar no solo estas cifras, sino la estructura tan delgada con la que alcanzó ProMéxico estos logros. Por citar un ejemplo, la oficina de ProMéxico en el Estado de Veracruz funcionaba con solo dos personas, y contribuyó en nueve años a que se realizaran 214 exportaciones por parte de empresas veracruzanas, por un monto total de 646 millones de dólares. Asimismo, la oficina en Veracruz apoyó para que se concretaran en el Estado 49 proyectos de inversión y reinversión por parte de compañías extranjeras, y que 11 empresas veracruzanas se internacionalizaran creando filiales en países extranjeros, para hacer más

eficientes y competitivas sus operaciones de negocios globales (ProMéxico, 2018, Cartera de proyectos de exportación e inversión).

Por resultados como los mencionados, en el año 2012 ProMéxico fue premiado por el Centro de Comercio Internacional (ITC) y la Conferencia de las Naciones Unidas para el Comercio y el Desarrollo (UNCTAD), dependientes de la ONU y la OMC, como la mejor agencia de promoción internacional de los países en desarrollo.

La principal debilidad de ProMéxico, y que el tiempo demostró que fue un riesgo real, fue la figura jurídica con que nació. Aunque el fideicomiso público fue muy versátil para su creación rápida, estaba expuesto, como todo fideicomiso, a ser extinguido cuando el Gobierno Federal así lo deseara.

Para tratar de evitar ese gran riesgo, la dirigencia de ProMéxico buscó en varias ocasiones su transformación a una figura que diera más certidumbre, como la de organismo público descentralizado, lo que implicaba una modificación a la Ley de Comercio Exterior o, mejor aún, la emisión de una nueva ley exprofeso que creara un nuevo organismo público descentralizado, intención que inclusive se le empezaba a llamar coloquialmente en el sector exportador como Ley ProMéxico.

Sin embargo, ambas alternativas implicaban un trabajo de concertación entre el poder ejecutivo y legislativo, a lo que el ejecutivo federal de los dos sexenios en los que existió ProMéxico, no le dio nunca la importancia que se debería, y la Ley ProMéxico nunca pasó más allá de una buena intención. 
En julio de 2018 fue electo presidente de la república López Obrador y, dados los temores entre exportadores e inversionistas, que originaban su postura a la apertura económica, diferente a la de los anteriores gobiernos, se pensó que una decisión sensata sería reforzar la actividad de ProMéxico, para dar mayor certidumbre a México ante la comunidad internacional, como destino seguro para inversiones productivas. Inclusive se llegaron a especular nombres de quien podría ser su próximo director general.

A inicios de septiembre de 2018 se hicieron públicas opiniones de quienes serían los futuros miembros del nuevo gabinete presidencial, unas a favor de mantener a ProMéxico y otros, expresando la idea de su desaparición, intención que cada vez empezó a cobrar mayor fuerza.

La intención parecía fuera de lugar, no parecía lógico desaparecer una institución que había dado tan buenos resultados, sobre todo en una etapa en la que se avecinaba cierta incertidumbre, y no tardaron en levantarse voces en contra de la idea, principalmente por parte de exportadores, inversionistas y casi la totalidad de las cámaras y organismos empresariales de México, mientras que fueron extremadamente pocas las figuras públicas y representantes económicos que se manifestaron a favor del cierre.

Para finales de septiembre de 2018, poco más de dos meses antes de su toma de posesión, el presidente López Obrador confirmó la intención de extinguir ProMéxico, opinando que era una institución que derrochaba recursos públicos y que era un exceso que hubiera un organismo con oficinas en el extranjero, cuando para ello estaban las embajadas. Igualmente anunció el cierre del Consejo de Promoción Turística de México, organismo que promovía en el extranjero a México como destino para el turismo extranjero, y del Instituto Nacional del Emprendedor.

Algo a resaltar, fue que, a pesar de la enorme cantidad de puntos de vista expresados en contra del cierre de ProMéxico, ninguna institución o representante empresarial fue más allá de las declaraciones ante los medios de comunicación. Nadie de entre los que defendían a ProMéxico realizó alguna acción legal o de protesta formal para detener el cierre anunciado. 
Una vez que inició la nueva administración federal, y ante la extinción anunciada, el sector exportador, inversionistas y el propio personal de ProMéxico esperaban que se fuera desarrollando una estrategia para substituir las actividades de promoción internacional que realizaba ProMéxico, a través de una nueva institución o alguna de las ya existentes, lo que podría llevar algunos meses o años.

Sin embargo, pronto se vio que ello no sería un proceso gradual, sino inmediato. La primera señal fue que, al concluir su gestión el último Director General de ProMéxico el 30 de noviembre de 2018, no se designó un nuevo director, dejándose en su lugar, solamente como encargado del despacho, al Jefe de la Unidad de Negocios Globales.

Menos de un mes después, el 26 de diciembre de 2018, y sin ningún aviso previo, los titulares de las oficinas de ProMéxico en la República recibieron una circular del encargado de la dirección, donde les instruyeron al cierre definitivo e irrevocable de las oficinas en un plazo de 5 días, el 31 de diciembre de ese mismo año. La misma suerte corrieron sus 46 oficinas en el extranjero, que unos días después también fueron cerradas en su totalidad (Cuevas L. M., comunicación personal, 2018).

Todo el personal de la institución fue liquidado, sin considerar el gran bagaje de capital humano en conocimientos y experiencia que se perdieron. Algunos de ellos fueron contratados posteriormente por otras dependencias federales o gobiernos locales, otros por empresas mexicanas o transnacionales, y algunos más se dedicaron a la consultoría privada (Cuevas L. M., comunicación personal, 2019).

Aunque ProMéxico dejó de realizar sus funciones el último día del 2018, fue hasta el 3 de mayo de 2019 cuando el presidente López Obrador emitió el decreto para la extinción legal del fideicomiso público ProMéxico (Decreto por el que se autoriza la desincorporación mediante extinción del Fideicomiso Público considerado Entidad Paraestatal denominado ProMéxico, 2019). Con ello concluyó una historia de éxito demostrada con evidencias objetivas; por ello, se insiste en la pregunta de hacer un replanteamiento a su desaparición.

Una de las principales críticas por parte de los sectores productivos de México que recibió la medida del cierre de ProMéxico, fue la ausencia de una estrategia gubernamental para 
realizar las actividades que llevaba a cabo la institución con el fin de atraer nuevas inversiones extranjeras y ampliar los mercados internacionales para las empresas del país, y el temor de que ello pudiera ocasionar afectaciones a la economía del país.

Esta idea fue reforzada posteriormente, al emitirse el Plan Nacional de Desarrollo 20192024, donde al tema de exportaciones y en general del comercio exterior; se le dedican sólo unas palabras en la sección III Economía, donde se menciona que "se alentará la inversión privada, tanto la nacional como la extranjera, y se establecerá un marco de certeza jurídica, honestidad, transparencia y reglas claras”.

Los temores no tardaron en hacerse realidad, y los flujos de inversión extranjera directa (IED) que habían pasado en 2009 de 17,853 millones de dólares anuales a 34,747 millones en 2018, prácticamente duplicándose durante la vida de ProMéxico, se redujeron en 1,834 millones de dólares en solo un año, para quedar en 2019 en 32,912 (Comisión Nacional de Inversiones Extranjeras, 2020, Informe estadístico sobre el comportamiento de la inversión extranjera directa en México enero-diciembre de 2019), y los datos parciales que se tienen de 2020, hacen prever una caída de la IED mucho más acelerada todavía. En el anexo 5 se presenta la estadística oficial de los montos de IED en México, emitida por la Comisión Nacional de Inversiones Extranjeras.

En el caso de las exportaciones de México, durante 2019 mantuvieron la inercia que había dejado la apertura de nuevos mercados realizada por ProMéxico, pero al eliminarse este impulso, y ante los problemas por la pandemia del COVID-19, en 2020 las ventas de productos mexicanos al exterior han tenido una grave caída. Los datos oficiales muestran que, en el primer semestre de 2018, cuando todavía funcionaba ProMéxico las exportaciones mexicanas no petroleras fueron de 203,844 millones de dólares; mientras que en el mismo periodo de 2019, se incrementaron ligeramente, un $4.8 \%$ con el impulso señalado, llegando a 213,536 millones de dólares (INEGI, 2020); pero, para el primer semestre de 2020, último dato semestral completo disponible, las exportaciones, se redujeron a 175,009 millones de dólares, un 14\% menos respecto al mismo lapso de 2018 y un $18 \%$ menos respecto a 2019. En el anexo 6 se presentan los montos de exportaciones mensuales en los años señalados publicados por INEGI. 
Aunque esa caída sin duda fue influida por la disminución de la demanda de bienes en muchos países por la pandemia de COVID-19, es probable que, de haberse mantenido ProMéxico se hubieran encontrado alternativas para colocar los productos mexicanos en otros mercados y que la caída no fuera tan pronunciada.

\section{METODOLOGÍA, TÉCNICAS Y MATERIALES EMPLEADOS}

El presente trabajo fue integrado mediante la investigación documental de la legislación aplicable, documentos emitidos por ProMéxico y de otros autores, referidos en las citas bibliográficas, así como puntos de vista de los autores.

\section{CONCLUSIONES}

Aunque lo ideal hubiera sido que la desaparición de ProMéxico no se hubiera dado, o se hubiera concretado hasta que se tuvieran perfectamente armadas estrategias para la promoción de México y de los productos mexicanos en el exterior, todavía puede ser momento para retomar el rumbo en ese sentido y replantear mecanismos que ayuden al país a no quedar rezagados en la carrera que libran los países para conquistar los nuevos mercados que se van abriendo y atraer inversionistas hacia destinos de inversión productiva. Esta competencia es cada vez más feroz y cada vez se suman nuevos países emergentes a ella, por lo que cualquier rezago prolongado en México en ese tema, puede hacer que el país pierda oportunidades de crecimiento que difícilmente se podrían volver a presentar.

Cabe señalar que, en este tiempo, ha habido ya intentos de retomar las funciones de ProMéxico por parte de diversas instituciones. Por ejemplo, la Secretaría de Economía, antigua cabeza de sector de ProMéxico, creó una Dirección General de Política de Promoción de Inversiones y Exportación, para realizar algunas de las funciones del extinto organismo, pero con un nivel jerárquico y tamaño estructura menor que el de ProMéxico, y sin la red de oficinas en México y el exterior que tenía el fideicomiso.

Igualmente, la Secretaría de Relaciones Exteriores ha tratado de realizar, a través de su red de embajadas y consulados, algunas de las funciones que realizaban las consejerías de 
ProMéxico, con magros resultados, dada la carencia que tienen de personal especializado en la promoción comercial y de inversiones, así como las limitaciones presupuestales que tienen.

Por su parte, algunos gobiernos estatales crearon sus propios organismos de promoción internacional. Una idea que puede ser buena, debido a que concentran sus esfuerzos en las áreas de oportunidad de esa entidad específica, pero que adolece de la fuerza que da una representación nacional ante gobiernos y otras instancias extranjeras.

Finalmente, algunos organismos del sector privado también han tratado de retomar funciones de ProMéxico, siendo el caso más notable el del Consejo Mexicano de Comercio Exterior, Inversión y Tecnología (COMCE), que ya desde antes tenía estrecha colaboración con ProMéxico, y después de su extinción siguió trabajando estrechamente con las embajadas mexicanas y gobiernos extranjeros.

\section{REFERENCIAS}

Comisión Nacional de Inversiones Extranjeras. (2020). Informe estadístico sobre el comportamiento de la inversión extranjera directa en México (enero-diciembre de 2019). Publicación de la CNIE.

Constitución Política de los Estados Unidos Mexicanos [CPEUM]. Art. 25. Ultima reforma 19 de febrero de 2021. (México).

De la Mora Luz María. (2017). Políticas para la atracción de inversión extranjera directa como impulsora de la creación de capacidades locales y del cambio estructural. CEPAL.

Decreto por el que se autoriza la desincorporación mediante extinción del Fideicomiso Público considerado Entidad Paraestatal denominado ProMéxico. 3 de mayo de 2019 DOF.

Decreto por el que se ordena la constitución del Fideicomiso Público considerado Entidad Paraestatal denominado ProMéxico. 1 de junio 2007 DOF.

Ley de Comercio Exterior. Ultima reforma 21 de diciembre de 2006 DOF.

Ley de Inversión Extranjera. Ultima reforma 15 de junio de 2018 DOF.

Ley que crea el Instituto Mexicano de Comercio Exterior. 31 de diciembre de 1970 DOF.

Plan Nacional de Desarrollo 2007-2012. 31 de mayo de 2007 DOF.

Plan Nacional de Desarrollo 2013-2018. 20 de mayo de 2013 DOF.

Plan Nacional de Desarrollo 2019-2024. 12 de julio de 2019 DOF.

ProMéxico. (2017). Revista Negocios ProMéxico Mayo/2017. Publicación de ProMéxico.

ProMéxico. (2017). Una década de promover a México en el mundo. Publicación de ProMéxico. 
ProMéxico. (2018). Cartera de proyectos de exportación e inversión. Publicación de ProMéxico.

Sección de comercio exterior del portal del Instituto Nacional de Estadística y Geografía (INEGI). (31 de mayo de 2020). www.inegi.org.mx . 
Anexo 1: Organigrama de ProMéxico

\begin{tabular}{|c|c|c|}
\hline \multirow{3}{*}{10 ìn } & \multirow{3}{*}{$\begin{array}{l}\text { MANUAL DE ORGANIZACIÓN } \\
\text { GENERAL DE PROMÉXICO }\end{array}$} & $\begin{array}{c}\text { FECHA DE EMISIÓN: } \\
03 / 08 / 2017 \\
\end{array}$ \\
\hline & & MOG PROMÉxICO \\
\hline & & Página: 31 de 142 \\
\hline
\end{tabular}

7. ORGANIGRAMA DE LA ESTRUCTURA ORGÁNICA BÁSICA

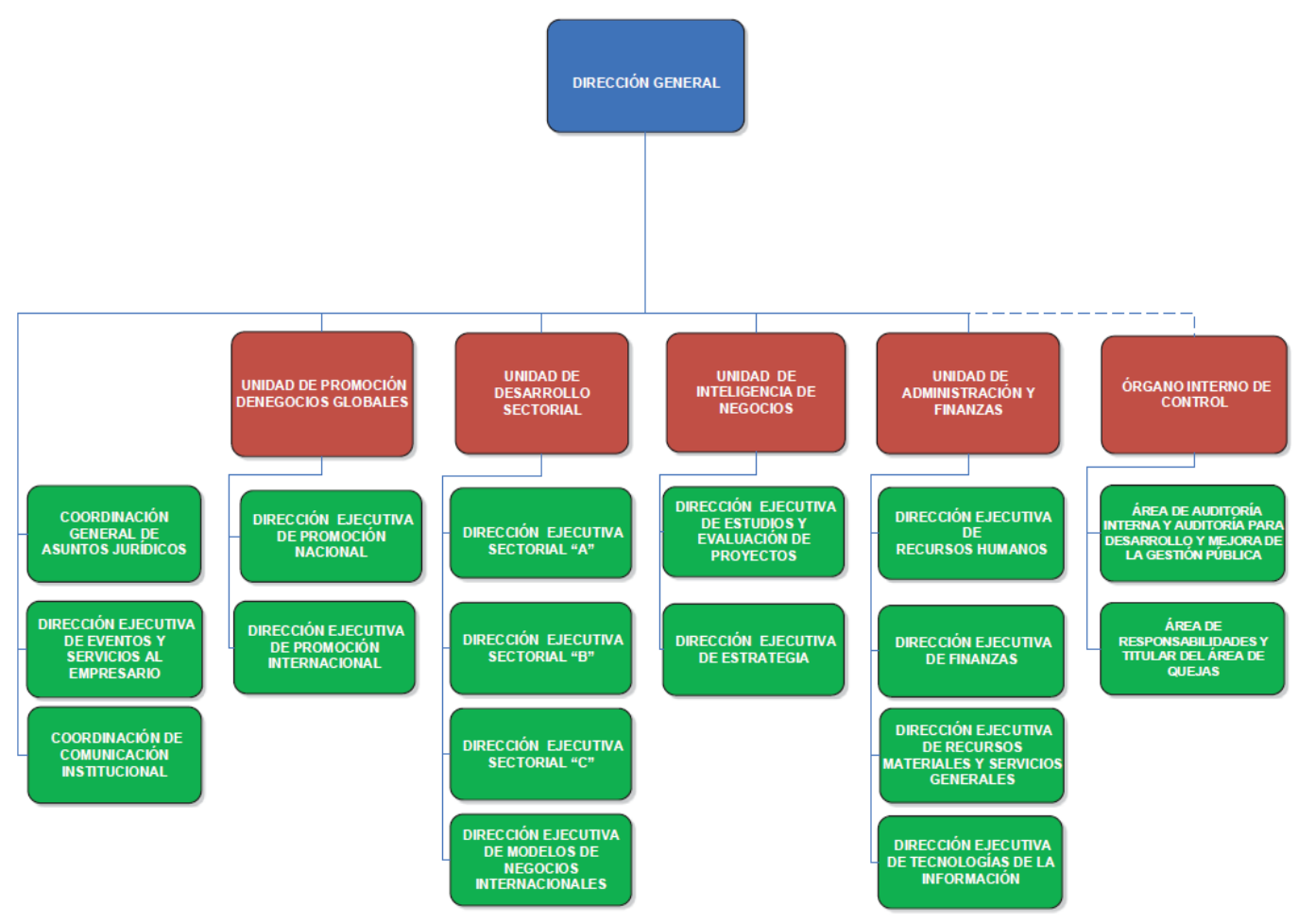

Cuevas L.M. \& Mariani R.

ProMéxico: una experiencia público-privada exitosa. su origen, consolidación, desaparición y potencial reimplantación. 
Anexo 2

CATALOGO DE APOYOS Y SERVICIOS DE PROMEXICO

\begin{tabular}{|c|c|c|}
\hline No. & APOYOS 2016 & $\begin{array}{c}\text { MONTO EN PESOS } \\
\text { MEXICANOS HASTA }\end{array}$ \\
\hline 1 & Asesoría en Empaque y Embalaje & 50,000 \\
\hline 2 & Asesoría Legal Internacional & 10,000 \\
\hline 3 & $\begin{array}{l}\text { Asesoría para la formación de Consorcios de Exportación } \\
\text { (REDEX) }\end{array}$ & 250,000 \\
\hline 4 & Asesoría Técnica en Procesos Productivos & 100,000 \\
\hline 5 & Centros de Distribución & 350,000 \\
\hline 6 & Certificaciones Internacionales & 100,000 \\
\hline 7 & Consultoría para Registro de Marca Internacional (IMPI) & 50,000 \\
\hline 8 & $\begin{array}{l}\text { Desarrollo de Estrategia de Ecommerce y Marketing } \\
\text { Digital }\end{array}$ & $50 \% \sin$ exceder 75,000 \\
\hline 9 & Ferias Tipo B & 750,000 \\
\hline 10 & Ferias Tipo C & 70,000 \\
\hline 11 & Formación de Ejecutivos en Comercio Exterior & 350,000 \\
\hline 12 & Misiones Estratégicas de Comercio & 50,000 \\
\hline 13 & $\begin{array}{l}\text { Programa de Promoción Internacional: Gerente de Redes } \\
\text { de Exportación (REDEX) }\end{array}$ & 360,000 \\
\hline No. & SERVICIOS 2016 & $\begin{array}{c}\text { TARIFA EN PESOS } \\
\text { MEXICANOS } \\
\text { MÁS IVA }\end{array}$ \\
\hline 1 & Agenda de Negocios & 15,000 \\
\hline 2 & Alianzas Estratégicas & Hasta $10,000,000^{*}$ \\
\hline 3 & Asesoría especializada & Hasta 5,000 \\
\hline 4 & Desarrollo de un Proyecto en Comercio Exterior & 78,000 \\
\hline 5 & Ferias Tipo A & Hasta $350,000^{*}$ \\
\hline No. & & TARIFA EN PESOS \\
\hline
\end{tabular}

Cuevas L.M. \& Mariani R. 


\begin{tabular}{|c|l|c|}
\hline & \multicolumn{1}{|c|}{ SERVICIOS 2016 } & $\begin{array}{c}\text { MEXICANOS } \\
\text { MÁS IVA }\end{array}$ \\
\hline 6 & Inteligencia Técnica para Exportadores & Hasta 150,000* \\
\hline 7 & Oferta Exportable en stand Institucional & Hasta 20,000* \\
\hline 8 & Pop Up Store/Showroom & Hasta 500,000* \\
\hline 9 & Presentación de Productos (degustación y/o cata) & Hasta 50,000* \\
\hline 10 & Programa de Capacitación en Comercio Internacional & Hasta $10,000^{*}$ \\
\hline 11 & Publicidad en medios & Hasta 275,000* \\
\hline
\end{tabular}

* La tarifa de los servicios dependerá primordialmente de la clasificación de la empresa de acuerdo a la Estratificación vigente de la Secretaría de Economía.

Anexo 3: PRESUPUESTO, LOGROS Y RENTABILIDAD DE PROMEXICO

Presupuesto otorgado a ProMéxico

Año Millones de MXN

2007

2008

2009

2010

2011

2012

2013

2014

2015

2016

2017

2018

Total
793

609

1,091

892

801

1,206

1,130

1,818

1,900

1,467

1,113

1,325

14,145
Logros 2007-2018

$\begin{array}{lrr} & \text { Millones de USD } & \text { Millones de MXN } \\ \text { Exportación } & 19,375 & 393,506 \\ \text { Inversión } & 124,181 & 2,522,116 \\ \text { Total } & 143,556 & 2,915,622\end{array}$

Tipo de cambio en noviembre de 2018:

20.31 MXN/USD

Rentabilidad (Logros/Gasto)

Exportación $\quad \mathbf{2 7 . 8}$

Inversión $\quad \mathbf{1 7 8 . 3}$

Total 206.1

Cuevas L.M. \& Mariani R. 
Horizontes de la Contaduría en las Ciencias Sociales, 74-97, Número 12, Enero-Julio 2020. ISSN: 2007-9796

Anexo 4: Cuenta pública de ProMéxico 2010-2018

\section{ECONOMÍA}

\section{CUENTA DE LA HACIENDA PÚBLICA FEDERAL DE 2010 \\ ANÁLISIS DEL EJERCICIO DEL PRESUPUESTO PROGRAMÁTICO DEVENGADO \\ PROMÉXICO}

\section{ANÁLISIS DEL GASTO POR CLASIFICACIÓN ECONÓMICA}

- En 2010, el presupuesto ejercido por el Fideicomiso ProMéxico fue de 980742.6 miles de pesos, cifra superior en 29.4 por ciento con relación a la asignación original. Este comportamiento, en términos de la clasificación económica del gasto, se debió principalmente al mayor ejercicio presupuestario en el rubro de Gastos de Inversión, (146.3 por ciento), derivado de la creación del Fondo Proaudiovisual.

> De los recursos erogados 891879.4 miles de pesos correspondieron a gasto directo, monto superior en 28.4 por ciento con relación a la asignación original, 67747.0 miles de pesos correspondieron a recursos propios cantidad mayor en 125.8 por ciento a la del presupuesto original y 21116.3 miles de pesos correspondieron a subsidios cantidad inferior en 36.8 por ciento a la del presupuesto original.

- El presupuesto ejercido con recursos propios fue resultado de los pagos realizados en apoyo a la realización de la Expo Shanghai 2010.

- El gasto ejercido con transferencias se debe en gran parte a la operación del Pabellón de México en la Expo Shanghai y la asignación del nuevo programa asignado a este Fideicomiso: Fondo Proaudiovisual y la organización del evento "Año de México en Francia".

\begin{tabular}{|c|c|c|c|c|c|c|c|c|}
\hline \multirow[b]{3}{*}{ Concepto } & \multicolumn{8}{|c|}{ Gasto Programable Devengado por Clasificación Económica de ProMéxico, 2010} \\
\hline & \multicolumn{3}{|c|}{ Presupuesto (Pesos) } & \multicolumn{2}{|c|}{ Variación Porcentual } & \multicolumn{3}{|c|}{ Estructura Porcentual } \\
\hline & Original & Modificado & Ejercido & Ejer./Orig. & Ejer./Modif. & Original & Modificado & Ejercido \\
\hline TOTAL & 758041763 & 980742617 & 980742617 & 29.4 & 0.0 & 100.0 & 100.0 & 100.0 \\
\hline GASTO CORRIENTE & 724616081 & 898426348 & 898426348 & 24.0 & 0.0 & 95.6 & 91.6 & 91.6 \\
\hline Servicios Personales & 404705472 & 384471459 & 384471459 & -5.0 & 0.0 & 53.4 & 39.2 & 39.2 \\
\hline Gastos de Operación & 288024151 & 481377158 & 481377158 & 67.1 & 0.0 & 38.0 & 49.1 & 49.1 \\
\hline -Materiales y Suministros & 3323964 & 2045016 & 2045016 & -38.5 & 0.0 & 0.4 & 0.2 & 0.2 \\
\hline - Servicios Generales & 284700187 & 479332142 & 479332142 & 68.4 & 0.0 & 37.6 & 48.9 & 48.9 \\
\hline \multicolumn{9}{|l|}{ Subsidios } \\
\hline Otros de Corriente & 31886458 & 32577731 & 32577731 & 2.2 & 0.0 & 4.2 & 3.3 & 3.3 \\
\hline GASTO DE INVERSIÓN & 33425682 & 82316269 & 82316269 & 146.3 & 0.0 & 4.4 & 8.4 & 8.4 \\
\hline \multicolumn{9}{|l|}{ Inversión Física } \\
\hline \multicolumn{9}{|c|}{ - Bienes Muebles e Inmuebles } \\
\hline \multicolumn{9}{|l|}{ - Obra Pública } \\
\hline \multicolumn{9}{|l|}{ - Otros de Inversión Física } \\
\hline Subsidios & 33425682 & 21116269 & 21116269 & -36.8 & 0.0 & 4.4 & 2.2 & 2.2 \\
\hline Otros de Inversión & & 61200000 & 61200000 & & 0.0 & & 6.2 & 6.2 \\
\hline
\end{tabular}

Fuente: Proméxico.

CUENTA DE LA HACIENDA PÚBLICA FEDERAL DE 2012

ANÁLISIS DEL EJERCICIO DEL PRESUPUESTO PROGRAMÁTICO

PROMÉXICO

\section{ANÁLISIS DEL GASTO POR CLASIFICACIÓN ECONÓMICA}

> En 2012, el presupuesto pagado de ProMéxico fue de 1205785.8 miles de pesos, cifra superior en 2.2\% con relación al presupuesto aprobado. Este comportamiento se debió principalmente al mayor ejercicio presupuestario en los rubros de Gastos de Operación (10.2\%), Servicios Personales (10.4\%), respectivamente.

\begin{tabular}{|c|c|c|c|c|c|c|c|c|}
\hline \multirow{2}{*}{ Concepto } & \multicolumn{3}{|c|}{ Presupuesto } & \multicolumn{2}{|c|}{ Variación Porcentual } & \multicolumn{3}{|c|}{ Es truc tura Porcentual } \\
\hline & Aprobado & Mo dific a do & Pagado & Pag./Aprob. & Pag./Modif. & Aprob. & Modif. & Pag. \\
\hline TOTAL & 1179554051 & 1205785824 & 1205785824 & 2.2 & 0.0 & 100.0 & 100.0 & 100.0 \\
\hline $\begin{array}{l}\text { Gas to corrie ute } \\
\text { Servicios personales }\end{array}$ & $\begin{array}{r}916519051 \\
374343707\end{array}$ & $\begin{array}{r}953025824 \\
413349429\end{array}$ & $\begin{array}{r}953025824 \\
413349429\end{array}$ & $\begin{array}{r}4.0 \\
10.4\end{array}$ & $\begin{array}{l}0.0 \\
0.0\end{array}$ & $\begin{array}{r}77.7 \\
317\end{array}$ & $\begin{array}{r}79.0 \\
34.3\end{array}$ & $\begin{array}{r}79.0 \\
34.3\end{array}$ \\
\hline Gasto de operación & 430281843 & 474276087 & 474276087 & 10.2 & 0.0 & 36.5 & 39.3 & 39.3 \\
\hline - Ma te nia les y suministros & 4344666 & 1390690 & 1390690 & .68 .0 & 0.0 & 0.4 & 0.1 & 0.1 \\
\hline - Servicios generales & 425937177 & 472885397 & 472885397 & 11.0 & 0.0 & 36.1 & 39.2 & 39.2 \\
\hline \multicolumn{9}{|l|}{ Subsidios } \\
\hline Otros de comente & 111893501 & 65400308 & 65400308 & -41.6 & 0.0 & 9.5 & 5.4 & 5.4 \\
\hline Gasto de inversion & 263035000 & 252760000 & 252760000 & -3.9 & 0.0 & 22.3 & 21.0 & 21.0 \\
\hline Inversion fisica & 13035000 & 2760000 & 2760000 & -78.8 & 0.0 & 1.1 & 0.2 & 0.2 \\
\hline - Bienes muebles, inmue bles e intangbles & 10035000 & 0 & 0 & -100.0 & & 0.9 & 0.0 & 0.0 \\
\hline - Inversion Pública & & & & & & & & \\
\hline - Otros de inversión física & 3000000 & 2760000 & 2760000 & -8.0 & 0.0 & 0.3 & 0.2 & 0.2 \\
\hline Subsidios & 250000000 & 250000000 & 250000000 & 0.0 & 0.0 & 21.2 & 20.7 & 20.7 \\
\hline Otros de muersión & 0 & 0 & 0 & & & 0.0 & 0.0 & 0.0 \\
\hline
\end{tabular}

Cuevas L.M. \& Mariani R.

ProMéxico: una experiencia público-privada exitosa. su origen, consolidación, desaparición y potencial 


\section{CUENTA PÚBLICA 2017}

\section{ANÁLISIS DEL EJERCICIO DEL PRESUPUESTO DE EGRESOS PROMÉXICO}

\section{Estado Analítico del Ejercicio del Presupuesto de Egresos por Clasificación Económica y por Objeto del Gasto}

> En 2017 el presupuesto pagado de ProMéxico fue de 1,112,909.2 miles de pesos, cifra superior en 5.9\% con relación al presupuesto aprobado. Este comportamiento se debió principalmente al mayor presupuesto pagado en el rubro de servicios personales (18.9\%) y en servicios generales (10.4\%), específicamente en la partida de exposiciones.

\section{Gasto Corriente}

> El Gasto Corriente pagado fue mavor al presupuesto aprobado en $9.1 \%$. Su evolución por rubro de gasto se presenta a continuación:

* En Servicios Personales se registró un mayor gasto pagado de $18.9 \%$ respecto al presupuesto aprobado, que se explica principalmente por la autorización de la ampliación líquida con apoyo del Sector para el pago de nómina del mes de diciembre para el personal eventual, así como por una adecuación presupuestal en el techo de ProMéxico para cumplir con el Decreto que establece las condiciones para el otorgamiento de aguinaldo 0 gratificación de fin de año correspondientes al ejercicio fiscal 2017.

* En el rubro de Gasto de Operación se registró un mayor gasto pagado de $9.0 \%$, en comparación con el presupuesto aprobado, por el efecto neto de los movimientos compensados y reducciones líquidas, realizados en los siguientes capítulos de gasto:

- En Materiales y Suministros se registró un menor gasto pagado en $33.9 \%$, en comparación con el presupuesto aprobado debido principalmente a los movimientos presupuestarios para dotar de recursos a la partida 39801 Impuesto sobre Nómina, que de origen presentó un déficit presupuestario

- El presupuesto pagado en Servicios Generales fue mayor en $10.4 \%$ respecto al presupuesto aprobado, que se explica principalmente por la ampliación de recursos provenientes del Sector Central para apoyar la participación de ProMéxico en diversas ferias como la Hannover Messe 2018 y Word Economic Forum DAVOS. En este evento se reúnen líderes de opinión, empresarios de alto nivel, jefes de estado y figuras internacionales de distintos ámbitos para discutir los principales temas de la agencia internacional. Una reducción al techo presupuestario derivado de un reintegro al Ramo 23 "Provisiones Salariales y Económicas" correspondientes al capítulo 2000 y 3000 por economías al cierre del ejercicio.

* En Subsidios no se presupuestaron recursos

* En Otros de Corriente menor gasto pagado de $43.1 \%$ respecto al presupuesto aprobado, lo que se explica principalmente por las adecuaciones con el fin de cubrir el déficit presupuestario en el pago del aguinaldo y Laudos.

\section{Pensiones y Jubilaciones}

$>$ No se presupuestaron recursos.

Cuevas L.M. \& Mariani R.

ProMéxico: una experiencia público-privada exitosa. su origen, consolidación, desaparición y potencial 
Horizontes de la Contaduría en las Ciencias Sociales, 74-97, Número 12, Enero-Julio 2020. ISSN: 2007-9796

\section{Cuenta PúBlica 2018}

\section{ANÁLISIS DEL EJERCICIO DEL PRESUPUESTO DE EGRESOS PROMÉXICO}

\section{ESTADO ANALITICO DEL EJERCICIO DEL PRESUPUESTO DE EGRESOS POR CLASIFICACIÓN ECONÓMICA Y POR OBJETO DEL GASTO}

> En 2018 el presupuesto pagado de ProMéxico (K2W) fue de 1,325,349.8 miles de pesos, cifra superior en $22.4 \%$ con relación al presupuesto aprobado. Este comportamiento se debió principalmente al mayor gasto pagado en los rubros de Servicios Personales (10.0\%); Gasto de Operación (11.2\%) y de Subsidios para Inversión (78.3\%).

\section{GASTO CORRIENTE}

> El Gasto Corriente pagado observó un incremento de $6.4 \%$ con relación al presupuesto aprobado. Su evolución por rubro de gasto se presenta a continuación:

* En Servicios Personales se registró un mayor gasto pagado de 10.0\% respecto al presupuesto aprobado, atribuible básicamente a los siguientes factores:

- Transferencia de recursos de los capítulos 2000, 3000 y 4000 a fin de cumplir con el decreto que establece el otorgamiento del aguinaldo 2018 .

- Apoyo de recursos del sector central a fin de cumplir con el decreto que establece el otorgamiento del aguinaldo 2018.

* En el rubro de Gasto de Operación se registró un presupuesto pagado mayor en $11.2 \%$, en comparación con el presupuesto aprobado, por el efecto neto de los movimientos compensados y reducciones líquidas, realizados en los siguientes capítulos de gasto:

- En Materiales y Suministros se registró un gasto pagado superior en $0.6 \%$, en comparación con el presupuesto aprobado, debido en gran medida al incremento en alimentos y utensilios, combustibles, materiales de construcción, refacciones y accesorios menores.

- El presupuesto pagado en Servicios Generales fue superior en $11.5 \%$ respecto al presupuesto aprobado, que se explica primordialmente por la ampliación de recursos para el evento de Hannover Messe 2018 y la obtención de ingresos excedentes del primer trimestre del año, los cuales se ejercieron para gastos en organización de eventos.

- La variación entre el presupuesto ejercido y el programado corresponde al capítulo 3000 Servicios Generales derivado de la falta de obtención de ingresos propios al cierre del periodo por 569.8 miles de pesos y economías provenientes de recursos propios por 799.8 miles de pesos este último monto forma parte de la disponibilidad final.

* En Subsidios no se presupuestaron recursos originalmente

* En Otros de Corriente se observó un gasto pagado menor en $44.1 \%$ respecto al presupuesto aprobado. Esta variación se explica esencialmente por el apoyo de recursos al capítulo 1000 para pago de aguinaldo.

\section{Anexo 5}

\section{Apéndice Estadístico}

5.1 IED en México por tipo de inversión



Cuevas L.M. \& Mariani R.

ProMéxico: una experiencia público-privada exitosa. su origen, consolidación, desaparición y potencial reimplantación. 
Horizontes de la Contaduría en las Ciencias Sociales, 74-97, Número 12, Enero-Julio 2020. ISSN: 2007-9796

Anexo 6: Exportaciones no petroleras de México 2018-2020

Cifras en millones de dólares USD

\begin{tabular}{|c|c|c|c|c|c|c|c|}
\hline \multirow[b]{2}{*}{ Periodo } & \multirow[b]{2}{*}{ Total } & \multirow[b]{2}{*}{ Agropecuarias } & \multirow[b]{2}{*}{ Extractivas } & \multicolumn{3}{|c|}{ Manufactureras } & \multirow[b]{2}{*}{$\begin{array}{l}\text { Total 1er } \\
\text { Semestre }\end{array}$} \\
\hline & & & & Total & Automotriz & Resto & \\
\hline \multicolumn{8}{|l|}{2018} \\
\hline Enero & $28,404.9$ & $1,603.8$ & 500.1 & $26,301.0$ & $8,680.2$ & $17,620.8$ & \\
\hline Febrero & $32,494.9$ & $1,524.8$ & 495.0 & $30,475.2$ & $11,426.6$ & $19,048.6$ & \\
\hline Marzo & $37,027.9$ & $1,779.7$ & 619.9 & $34,628.3$ & $13,249.3$ & $21,379.0$ & \\
\hline Abril & $34,555.4$ & $1,575.2$ & 589.0 & $32,391.2$ & $11,552.8$ & $20,838.3$ & \\
\hline Mayo & $36,386.9$ & $1,588.6$ & 546.4 & $34,251.8$ & $11,347.0$ & $22,904.8$ & \\
\hline Junio & $34,974.3$ & $1,243.5$ & 546.4 & $33,184.4$ & $12,054.0$ & $21,130.4$ & $203,844.3$ \\
\hline Julio & $34,135.7$ & $1,052.8$ & 512.9 & $32,570.0$ & $11,069.1$ & $21,500.9$ & \\
\hline Agosto & $36,805.4$ & $1,026.6$ & 510.5 & $35,268.3$ & $12,567.4$ & $22,701.0$ & \\
\hline Septiembre & $34,932.6$ & 901.4 & 522.1 & $33,509.1$ & $12,890.7$ & $20,618.4$ & \\
\hline Octubre & $38,737.3$ & $1,266.0$ & 407.9 & $37,063.3$ & $13,215.1$ & $23,848.2$ & \\
\hline Noviembre & $36,286.2$ & $1,373.1$ & 426.5 & $34,486.5$ & $12,580.1$ & $21,906.4$ & \\
\hline Diciembre & $35,341.9$ & $1,572.0$ & 555.1 & $33,214.7$ & $11,546.2$ & $21,668.6$ & \\
\hline \multicolumn{8}{|l|}{2019} \\
\hline Enero & $30,545.9$ & $1,634.4$ & 361.9 & $28,549.5$ & $9,324.6$ & $19,224.9$ & \\
\hline Febrero & $33,828.7$ & $1,589.4$ & 515.2 & $31,724.1$ & $11,621.8$ & $20,102.3$ & \\
\hline Marzo & $36,676.0$ & $1,741.5$ & 591.5 & $34,342.9$ & $13,290.9$ & $21,052.0$ & \\
\hline Abril & $37,327.8$ & $1,921.3$ & 461.2 & $34,945.3$ & $12,594.9$ & $22,350.4$ & \\
\hline Mayo & $39,182.4$ & $1,618.4$ & 587.4 & $36,976.6$ & $13,162.2$ & $23,814.4$ & \\
\hline Junio & $35,975.8$ & $1,321.5$ & 506.4 & $34,148.0$ & $12,580.6$ & $21,567.3$ & $213,536.5$ \\
\hline Julio & $36,915.2$ & $1,237.8$ & 547.5 & $35,130.0$ & $12,465.5$ & $22,664.5$ & \\
\hline Agosto & $38,143.0$ & $1,062.7$ & 517.4 & $36,562.9$ & $13,786.0$ & $22,777.0$ & \\
\hline Septiembre & $35,289.3$ & $1,034.0$ & 469.3 & $33,785.9$ & $12,534.0$ & $21,251.9$ & \\
\hline Octubre & $38,928.5$ & $1,374.3$ & 524.9 & $37,029.3$ & $12,397.7$ & $24,631.6$ & \\
\hline Noviembre & $35,626.9$ & $1,548.7$ & 519.3 & $33,558.9$ & $12,138.4$ & $21,420.5$ & \\
\hline Diciembre & $36,422.4$ & $1,755.6$ & 586.7 & $34,080.0$ & $11,860.0$ & $22,220.0$ & \\
\hline \multicolumn{8}{|l|}{2020} \\
\hline Enero R & $31,380.0$ & $1,794.8$ & 478.7 & $29,106.4$ & $10,211.6$ & $18,894.8$ & \\
\hline Febrero & $34,946.5$ & $1,702.1$ & 513.1 & $32,731.3$ & $12,014.8$ & $20,716.5$ & \\
\hline Marzo & $37,097.0$ & $2,063.1$ & 705.6 & $34,328.3$ & $12,621.9$ & $21,706.3$ & \\
\hline Abril & $22,626.8$ & $1,763.6$ & 553.2 & $20,310.0$ & $2,627.3$ & $17,682.7$ & \\
\hline Mayo & $17,140.4$ & $1,479.4$ & 396.1 & $15,264.9$ & $1,307.4$ & $13,957.6$ & \\
\hline Junio 0 & $31,818.5$ & $1,737.9$ & 553.4 & $29,527.2$ & $8,683.4$ & $20,843.8$ & $175,009.2$ \\
\hline \multicolumn{8}{|c|}{${ }^{{ }^{\circ} \text { Cifras oportunas a }}$} \\
\hline${ }^{R}$ Cifras revisadas & & & & & & & \\
\hline
\end{tabular}

Cuevas L.M. \& Mariani R.

ProMéxico: una experiencia público-privada exitosa. su origen, consolidación, desaparición y potencial reimplantación. 